\title{
LTE-Advanced Self-Organising Network Conflicts and Coordination Algorithms
}

\author{
Hafiz Yasar Lateef ${ }^{1}$, Member IEEE, Ali Imran ${ }^{2}$, Member IEEE, Muhammad Ali Imran ${ }^{3}$, Senior Member IEEE, \\ Lorenza Giupponi ${ }^{4}$, Senior Member IEEE, Mischa Dohler ${ }^{5}$, Fellow IEEE \\ 1 Qatar Mobility Innovations Center (QMIC), Doha, Qatar \\ 2 University of Oklahoma, Tulsa, 74135, USA \\ 2 CCSR, University of Surrey, Guildford, United Kingdom. GU2 7XH. \\ 3 Centre Tecnolgic de Telecomunicacions de Catalunya (CTTC), \\ Av. Carl Friedrich Gauss 7, Barcelona, Spain 08860 \\ 4 King's College London, London, United Kingdom \\ Email: yasarl@qmic.com; ali.imran@ou.edu; m.imran@surrey.ac.uk; lorenza.giupponi@cttc.es; \\ mischa.dohler@kcl.ac.uk
}

Abstract - Self-Organising Network (SON) functions have been introduced in the Long Term Evolution (LTE) and LTE-Advanced standards by the Third Generation Partnership Project (3GPP) as an excellent solution that promises enormous improvements in the network performance. However, the most challenging issue in implementing SON functions in reality is the identification of the best possible interactions among simultaneously operating and even conflicting SON functions in order to guarantee a robust, stable and desired network operation. In this direction, the first step is the comprehensive modelling of various types of conflicts among SON functions, not only to acquire a detailed view of the problem, but also to pave the way for designing appropriate Self-Coordination mechanisms among SON functions. In this paper we present a comprehensive classification of SON function conflicts, which leads the way for designing suitable conflict resolution solutions among SON functions and implementing SON in reality. Identifying conflicting and interfering relations among autonomous network management functionalities is a tremendously complex task. We demonstrate how analysis of fundamental trade-offs among performance metrics can lead us to the identification of potential conflicts. Moreover, we present analytical models of these conflicts using Reference Signal Received Power (RSRP) plots in multi-cell environments which help to dig into the complex relations among SON functions. We identify potential chain reactions among SON function conflicts that can affect the concurrent operation of multiple SON functions in reality. Finally, we propose a Self-Coordination framework for conflict resolution among multiple SON functions in LTE/LTE-Advanced networks, while highlighting a number of future research challenges for conflict-free operation of SON.

Index Terms-Self-Organising Network (SON), Long Term Evolution (LTE), Self-Coordination, SON conflicts, Self-Optimisation

\section{INTRODUCTION}

Oiv iven that network densification is emerging as the dominant capacity enhancement theme in both existing and future cellular networks, operational complexity and Operational Expenditures (OPEX) are bound to increase. Another, challenging feature of the future cellular landscape is that, more and more network nodes will be deployed impromptu. Consequently, Self-Organising Network (SON) functions are being considered as essential feature to ensure the technical as well 
as financial viability of emerging and future cellular networks. This is reflected by identification of key SON use cases by Next Generation Mobile Networks (NGMN) [1] and their ongoing standardization by Third Generation Partnership Project (3GPP). As a result of the stark demand from operators, and endorsements by standardization body, research on developing SON functions for the use cases identified by NGMN and 3GPP has gained significant momentum both in academic and industrial circles. For a detailed review of the state-of-the-art SON functions reader is referred to $[2,3]$.

Although many individual SON solutions for each NGMN use case promise gain with respect to its intended Key Performance Indicators (KPI) as discussed in [2], different SON functionalities can however have a range of conflicts when working concurrently in the same network. Such conflicts may compromise the overall gain of SON and may also undermine system stability. Since networks are becoming denser and more heterogeneous and SON functionalities have to be exploited at wide scale, the analysis of potential conflicts generated by the numerous autonomous functionalities in heterogeneous networks can be extremely challenging. Therefore, Self-Coordination among conflicting SON functions is essential in order to enable a stable network operation with tight operator control over the network behaviour. Self-Coordination among conflicting SON functions is thus understandably endorsed by the 3GPP System Architecture (SA) group (SA5) in 3GPP Release-11 [4].

Despite its significance and implications, the problem of self-coordination has not been deeply investigated in literature. We highlight here the main contributions. The Socrates project team took the first step in this direction and organized selected interrelated parameters which could cause parametric conflict into basic groups [5]-[7]. Initial challenges on characteristics, parametric, and measurement conflicts when integrating SON functions into next generation wireless networks were then described in [8]. However, none of these works provide comprehensive identification, annotation and classification of SON function conflicts. Some Self-Coordination mechanisms for SON are described in [9]. However, [9] does not identify the application of these Self-Coordination mechanisms for specific SO function conflicts. In [10], for the first time authors have identified and categorised SON function conflicts, based on different criteria such as the network deployment, the Key Performance Indicators, the measurement and logical dependencies, the output parameters' direction/magnitude, etc. This is a very interesting first step towards self-coordination, however, the resolution of SON conflicts requires a deeper analysis, modeling and classification, as we will describe in the rest of the paper.

Until now the state-of-the-art work on SON conflict classification has mainly focused on hard classification where each SON conflict is categorized into exactly one category while ignoring the fact that a SON conflict may arise due to more complicated interactions deriving from more than one conflicting conditions. In this case, it is not optimal to classify a conflict in a certain category, but it is better to work considering that any conflict may have a fuzzier classification, with different degrees of membership to various categories, depending upon the cause of the conflict. We will refer in this paper to this novel kind of classification as soft classification. The overall soft and hard classification of conflicts will be 
referred to as Hybrid classification. In this paper, we provide a comprehensive hybrid classification of SON function conflicts. It is worth mentioning here that the proposed hybrid classification of SON function conflicts paves the way for not only to identify the root cause of the conflicts but also to design efficient self-coordination solutions. We also propose another unique SON conflict classification called Nested Conflicts. Specifically, some of the SON function conflicts may occur as a reaction of other SON function conflicts. We categorise chain reactions among SON function conflicts in Nested Conflicts category. To the best of authors' knowledge, Hybrid classification of SON conflicts has never been proposed before.

In order to overcome the tremendous difficulty of identifying the potential conflicts in the complex and intricate future heterogeneous network scenarios, we propose and demonstrate a method to analytically identify potential conflict based on fundamental trade-offs already well known in cellular network literature. We also present another method to model the SON function conflicts using 3GPP handover triggering event equations and Reference Signal Received Power (RSRP) plots in multicell environments. Building on these developments, we outline a hybrid Self-Coordination scheme and we provide mechanisms for conflict-free operation of SON functions. The conflict identification schemes and the coordination mechanisms provided in this paper are generally valid for range of SON function conflicts. However, in order to provide insights into the addressed problem, in different sections we will discuss specific SON function conflict examples as proof of concepts.

The rest of the paper is organized as follows. Analytical modelling of SON function conflicts is presented in Section II. A Hybrid classification of SON function conflicts and chain reactions among SON function conflicts are presented in Section III. A Hybrid Self-Coordination mechanism based upon 3GPP architecture is proposed in Section IV. The performance evaluation of the proposed Hybrid Self-Coordination solution is also presented in Section IV. Finally, conclusion and future work are presented in Section V.

Notation: ANR: Automatic Neighbour Relation, CCO: Coverage and Capacity Optimisation, CIO: Cell Individual Offset, COC: Cell Outage Compensation, CQI: Channel Quality Indicator, EE: Energy Efficiency, eNB: Evolved Node B, HeNB: Home eNB, ICIC: Inter-Cell Interference Coordination, KPI: Key Performance Indicator, MLB: Mobility Load Balancing, MRO: Mobility Robustness Optimisation, NTM: Network Topology Mutation, OAM: Operation, Administration and Management, PCI: Physical Cell Identity, RAT: Radio Access Technology, RET: Remote Electrical Tilt, RLF: Radio Link Failure, RSRP: Reference Signal Received Power, RSRQ: Reference Signal Received Quality, SO: Self-Organising, TXP: Transmission Power, and UE: User Equipment. 


\section{Analytical Modelling of Self-Organising Function Conflicts}

The identification of potential conflicts among miscellany of SON functions in a cellular heterogeneous network which might be operating concurrently to optimize same network parameters and metrics at different spatial and temporal scales, is extremely complex [2]. In order to find a proper approach for anticipation and subsequent mitigation of these conflicts it is first essential to understand the nature and origin of the potential conflicts. Exploitation of the fundamental knowledge of the trade-offs between several desirable performance metrics, is one possible approach for identifications of underlying conflicts as we will show through an analysis of Energy Efficiency (EE) and spectral efficiency trade-off [11, 12]. In the following we first provide an example of how to identify potential conflicts from the analytical perspective. Then, we devote two subsections to the analytical description of the generation of complicated conflicts, among different SON functions, based on the analysis of RSRP curves.

\section{A. Mapping of Conventional Cellular Network Trade-offs to SON Function Conflicts}

As explained above, theoretical trade-offs in communication networks can be used as a guide for the identification of potential conflicts in future cellular networks. If the optimization or configuration of future cellular networks is done in a Self-Organized manner, then these trade-offs directly result in SON function conflicts. One of these trade-offs is between the energy and spectral efficiency and this maps directly to Coverage and Capacity Optimisation (CCO) and EE SON functions conflict.

To analyse the underlying interplay of CCO and EE SON functions conflict we can consider a simplified scenario of a single link transmission over Additive White Gaussian Channel (AWGN) with bandwidth $W$. In order to achieve a rate $R$, with transmit power $P$, the noise power spectral density $N_{0}$, and energy efficiency represented by $E_{J}$ (in terms of bit/Joule), we can show that the relationship between energy efficiency and spectral efficiency $R / W$ is given by [11]:

$$
E_{J}=\frac{1}{N_{0}}\left(\frac{R / W}{2^{R} / W-1}\right)
$$

It is evident from equation (1) that increasing the spectral efficiency decreases the energy efficiency, since the denominator increases exponentially. Although this equation shows a fundamental limit on the trade-off between two desirable objectives, this gives a solid reason to believe that $\mathrm{EE}$ and $\mathrm{CCO}$ functions in SON may suffer from a conflict and require a framework to manage this conflict. More specifically, EE function may try to reduce the Evolved Node B (eNB) Transmission Power (TXP) or try to activate the sleep mode at eNB for energy saving, while CCO function may try to increase TXP for better coverage and capacity. Hence, both EE and CCO may try to set different values for TXP, which consequently causes an output parameter conflict.

\section{B. Case Study of Mobility Load Balancing (MLB), EE and CCO Function Conflicts}

The conflict among MLB, CCO and EE functions is modeled with the help of RSRP plot in multi-cell environments as shown in Fig. 1 (a). More specifically, the X-axis and Y-axis represent the distance and RSRP respectively. The User Equipments (UEs) move between eNB1 and eNB2. The MLB function monitors the load of cells periodically and adjusts the handover region by 
biasing the handover parameter, i.e., cell specific offset of the neighbour cell $\left(O_{c n}\right)$ when the traffic load is unbalanced. The handover triggering condition (A3) defined by 3GPP [13]-15] as "Neighbour becomes offset better than primary cell" is mathematically described as follows:

$$
M_{n}>M_{p}+\left(H-O_{c n}\right)
$$

where $H$ is the hysteresis parameter for handover and $M_{n}, M_{p}$ represent the RSRP without offset of primary and neighbour cells, respectively. The frequency specific offsets are set to zero in equation (2).

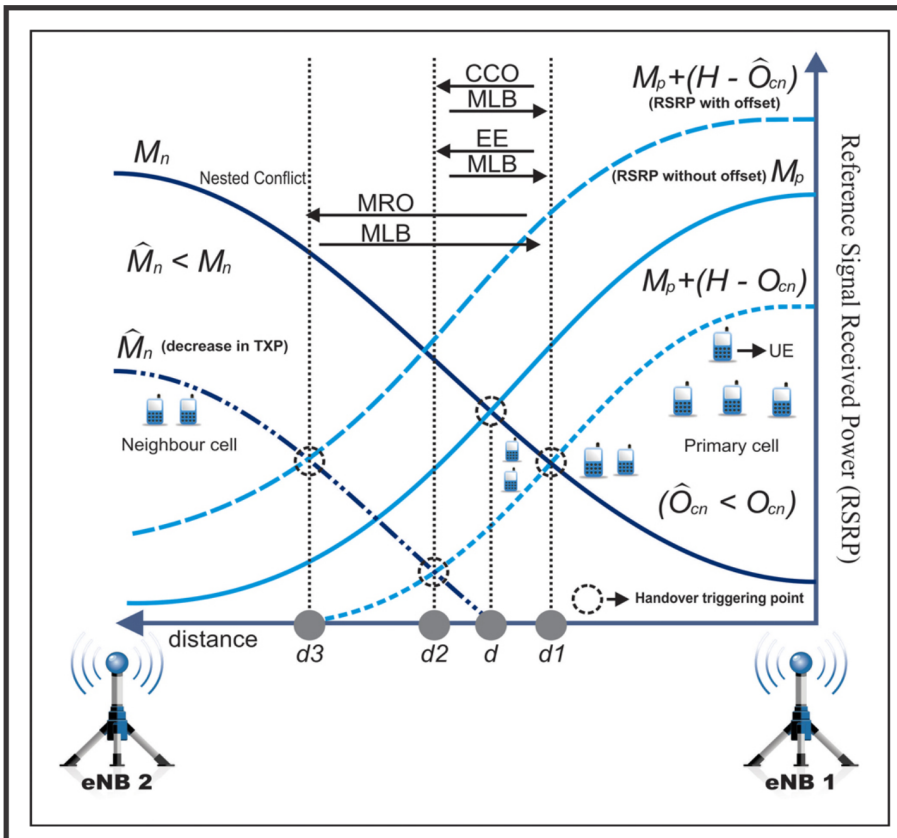

(a)

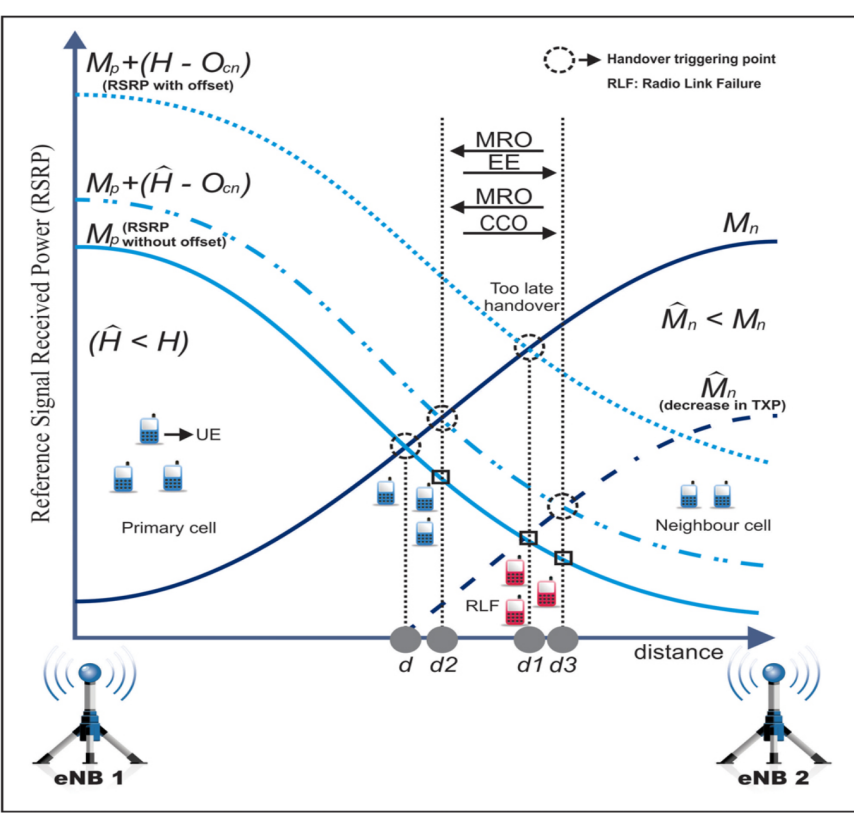

(b)

Fig. 1 (a) RSRP plot for illustration of conflict between MRO, EE and CCO SON Functions in Multi-cell environments (b) RSRP plot for illustration of conflict between MLB, CCO, EE and MRO SON Functions in Multi-cell environments

If the primary cell is heavily loaded and its neighbouring cell is lightly loaded, then the MLB function of the primary cell increases $O_{c n}$ to make the handover triggering earlier at $d_{1}$ instead of its original handover triggering point at $d$ in order to steer traffic towards the neighbouring cell as shown in Fig. 1 (a). It must be mentioned here that in practical scenarios $O_{c n}$ can have values between $-6 \mathrm{~dB}$ to $6 \mathrm{~dB}$ [8]. However, $\mathrm{CCO}$ or EE function of the neighbouring cell may decide to decrease the antenna TXP, due to its light load condition. This will shift the RSRP curve of the neighbouring cell to the case $\widehat{M}_{n}$ indicated on the graph for the reduced TXP. As a result, the new handover triggering point delays to position $d_{2}$ instead of earlier triggering position $d_{1}$ and consequently causes a conflict with the objective of the MLB function. Moreover, the weak received signal strength at the handover triggering position $d_{2}$ may cause a call drop or Radio Link Failure (RLF) and consequently triggers the 
Mobility Robustness Optimisation (MRO) function. In order to avoid handover problems, the MRO function should decrease the cell specific offset of the neighbour cell to $\hat{O}_{c n}\left(\hat{O}_{c n}<O_{c n}\right)$, which shifts the handover triggering position to $d_{3}$ as shown in Fig.

1 (a). However, the primary cell is still heavily loaded and the corresponding MLB function of the primary cell would increase the cell specific offset of the neighbour cell to $O_{c n}$, while MRO may try to decrease $O_{c n}$ again due to the handover problems. This situation will trigger a conflict between MRO and MLB functions, which will ultimately generate oscillations of network parameter's configuration. It must be mentioned here that the MRO and MLB functions conflict is triggered by MLB and CCO or MLB and EE functions conflict.

\section{Case Study of MRO, EE and CCO Function Conflicts}

This conflict between MRO, EE and CCO SON functions is modeled with the help of the RSRP plot in multi-cell environments as shown in Fig. 1 (b). The MRO function monitors handover problems, such as too late handover, ping pong etc. In connected mode handover, decisions are made at eNBs by using UEs measurement reports. The eNBs configure triggering points for UE measurement reports, in such a way that these reports are sent when certain conditions are fulfilled. For example, equation (2) describes a specific handover triggering condition (A3). If frequency specific offsets are zero, then the handover triggering point is calculated by setting hysteresis parameter $H$ and cell specific offset for neighbour cell $O_{c n}$. Hysterisis parameter $H$ can have 21 values between 0 and $10 \mathrm{~dB}$ with a step size of $0.5 \mathrm{~dB}[13,14]$. If hysteresis parameter is set to a large value, then the handover triggering point is configured such that it causes too late handover at position $d_{1}$, as shown in Fig. 1 (b). Too late handover can cause RLF due to weak RSRP at position $d_{1}$. In case of RLF, the MRO function decreases the handover hysteresis to $\widehat{H}(\widehat{H}<H)$ in order to shift the handover triggering point to some earlier position $d_{2}$ as shown in Fig. 1 (b). However, if EE or CCO function in the neighbouring cell decreases its transmission power due to light load conditions, then the RSRP curve of the neighbouring cell shifts to the case $\widehat{M}_{n}$, thus further delaying the handover triggering point to position $d_{3}$ as shown in Fig. 1 (b). As a result, the MRO function objective is in conflict with EE or CCO function.

\section{Hybrid Classification Of Self-Organising Network Function Conflicts}

In this section, we provide a comprehensive taxonomy and classification of conflicts among SON functions. We first present a hard classification of different kinds of conflicts that may be generated in LTE/LTE-Advanced Self-Organising networks. Then on, we present soft classification of conflicts among SO functions. We also present nested conflicts in this section. 


\section{A. Hard Classification of SON Function Conflicts}

Most recently, in [10], Lateef et al. presented a hard classification framework for SON function conflicts based on five major categories: Parameter conflicts, Network Topology Mutation conflicts, Key Performance Indicator conflicts, Logical Dependency conflict and Measurement conflict.

1) Parameter conflicts can be further classified into output and input parameter conflicts. Output Parameter conflicts may occur when two or more SON functions try to modify the same network configuration parameter. Input Parameter conflict may arise when a SON function is triggered by an input parameter whose value is dependent upon some other network parameters.

2) Network Topology Mutation conflict may occur due to the change in network conditions by the addition or removal of relay, eNB or Home eNB (HeNB).

3) Key Performance Indicator conflict may arise when different SON function actions try to alter the same KPI of a cell, while adjusting different network configuration parameters.

4) Measurement conflict may happen if a SON function is either triggered or computes new parameter configuration values based on outdated measurements.

5) Logical Dependency conflict may take place if there is a logical dependency among the objectives of SON functions. The interested reader may refer to [10] for further details on this hard classification of conflicts.

\section{B. Soft Classification of SON Function Conflicts}

We analyze here the potential interference that may exist among different kinds of conflicts defined in the above subsection.

1) Some of the SON function conflicts associated with the Output Parameter conflict category may also be classified in Measurement and Logical Dependency conflict categories. More specifically, if an outdated measurement activates a SON function which computes contradictory configuration settings for network parameters, as compared to other SON functions, then this kind of conflict belongs to both Output Parameter and Measurement conflict categories. Moreover, if some of the conflicting SON functions not only compute contradictory configuration settings for network parameters as compared to other SON functions, but also have logical dependency between their objectives, then these kinds of conflicts belong to both Output Parameter and Logical Dependency conflict categories. A representative example of specific SON functions conflict association with Output Parameter, Measurement and Logical Dependency conflicts is presented below.

CCO and Inter-Cell Interference Coordination (ICIC) Functions Conflict: Fig. 2 depicts the effect that this conflict can have on the cell boundaries, as well as the message exchanges at architecture level through the Itf-S and the X2 interfaces. ICIC functionalities are typically implemented distributively while CCO functions typically use a centralized architecture located in the Operation, Administration and Management (OAM). For centralized implementation of CCO function, signaling and KPIs are exchanged with eNBs over the Itf-S interface. On the other hand, the UE reports on measurements related to RSRP, 

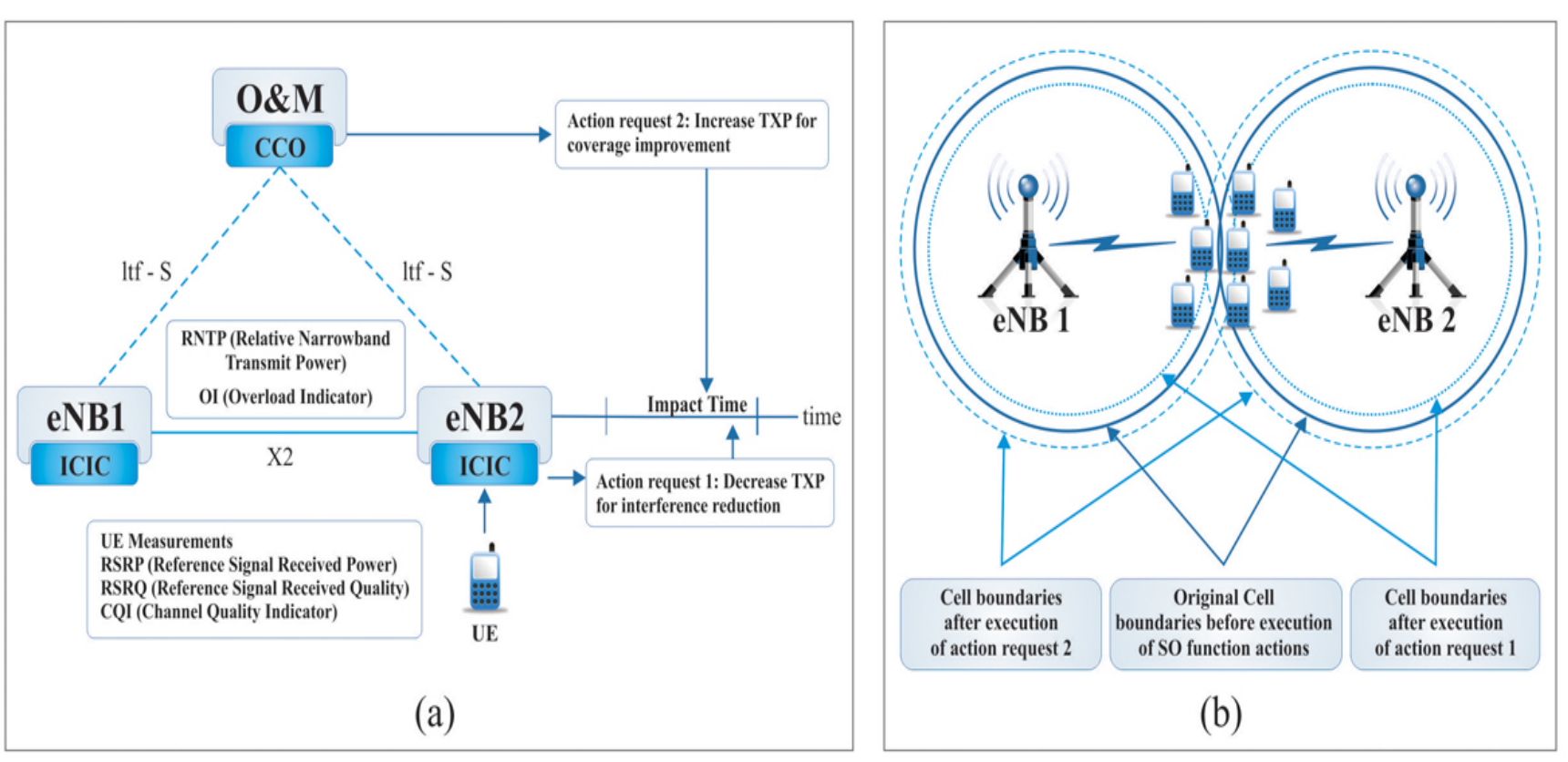

Fig. 2. Conflict between CCO \& ICIC SON functions: (a) 3GPP architecture model for CCO \& ICIC SON functions conflict (b) Impact of CCO \& ICIC SON functions conflict on cell boundaries.

Reference Signal Received Quality (RSRQ) and Channel Quality Indicator (CQI), and X2 signaling in terms of Relative Narrowband Transmit Power (RNTP) and Overload Indicator (OI) supports a dynamic ICIC coordination among cells. However, outdated measurement reports of CQI or RSRQ may cause false triggering of ICIC function. As a result, ICIC function may try to decrease TXP for interference reduction, while CCO function may try to increase TXP for coverage improvement which can not only cause output parameter configuration conflict but also measurement conflict.

In another case, the CCO function may try to modify the antenna Remote Electrical Tilt (RET) for coverage improvement while the ICIC function may try to change TXP for interference reduction. In this scenario, both CCO and ICIC functions configure different network parameters with different objectives. This may negatively affect the performance gains made by each function, as both of them affect the same coverage area and have an implicit relationship between their objectives. As a result, CCO and ICIC functions trigger a Logical Dependency conflict.

2) SON function conflicts associated with Logical Dependency conflict category may also be classified in Measurement conflict category. More specifically, if an outdated measurement value triggers a SON function conflict and there is also a Logical Dependency between the objectives of these conflicting SON functions, then this type of conflict belongs to both Measurement and Logical Dependency conflict categories. A representative example of specific SON functions conflict association with both Logical Dependency and Measurement conflicts is presented below.

Mobility Robustness Optimisation (MRO) and CCO Functions Conflict: CCO function can modify RET in order to optimize coverage and capacity, which will have impact on the cell size. Meanwhile, if a MRO function is triggered based on 
measurements collected in the time before the change in cell size is reflected in measurements, then the MRO function could be using outdated measurements for calculating new handover settings. As a result, MRO and CCO functions may degrade the performance improvement achieved by each other and cause a Measurement conflict.

In another scenario, a MRO function can adjust Cell Individual Offset (CIO) and hysteresis parameter in order to calculate the optimum handover triggering point. However, the CCO function in the neighbouring cells can modify the RET in order to improve coverage and capacity, which will change coverage overlap between these cells and hence the hysteresis region. As a result, the handover triggering point calculated by the MRO may become non-optimum due to inter-relationship between the objectives of MRO and CCO functions. This kind of conflict is a Logical Dependency conflict.

3) SO function conflicts associated with Input Parameter conflict category may also be classified in the Measurement conflict category. More specifically, if an outdated measurement value on the one hand triggers a SON function conflict and on the other hand this measurement serves as input parameter to the conflicting SON functions, then this type of conflict belongs to both Measurement and Input Parameter conflict categories. A representative example of specific SON functions conflict association with both Input Parameter and Measurement conflicts is presented below.

Conflict between two Physical Cell Identity (PCI) Functions: In order to allocate PCI to the target cell, PCI configurations of neighbouring cells are gathered as an input to PCI function. However, if there are two PCI functions executing with intersecting impact area and time, then the PCI configurations gathered by the first PCI function could be modified by the second PCI function while the first PCI function is allocating physical cell identity to the target cell. Hence, the new physical cell identity by the first PCI function might be erroneous due to outdated input neighbouring configurations. In this example, PCI configurations of neighbouring cells are acting as both measurement and the input value to the PCI function. Therefore, this kind of conflict is associated with both input parameter and measurement conflict categories.

4) SON function conflicts associated with Network Topology Mutation conflict category may also be classified in Measurement conflict category. More specifically, if an outdated measurement value triggers a SON function conflict and these conflicting SON functions are also affected by the addition or removal of network nodes, then this type of conflict belongs to both Measurement and Network Topology Mutation conflict categories. Similarly, SON function conflicts associated with Key Performance Indicator conflict category may also be linked with Measurement conflict category. More specifically, if an outdated measurement value triggers a SON functions conflict and these conflicting SON functions also affect the same KPI of a cell, then this type of conflict belongs to both the Measurement and Network Topology Mutation conflict categories.

A comprehensive list of soft classification of SON functions conflicts is presented in TABLE 1. 
TABLE 1

SOFT CLASSIFICATION OF SON FUNCTION CONFLICTS

\begin{tabular}{|c|c|c|c|c|c|c|c|}
\hline $\begin{array}{l}\text { Sr. } \\
\text { No }\end{array}$ & $\begin{array}{l}\text { SON function } \\
\text { conflict scenario }\end{array}$ & $\begin{array}{l}\text { Output } \\
\text { Parameter } \\
\text { conflict }\end{array}$ & $\begin{array}{c}\text { Logical } \\
\text { Dependency } \\
\text { conflict }\end{array}$ & $\begin{array}{c}\text { KPI } \\
\text { conflict }\end{array}$ & $\begin{array}{l}\text { Measurement } \\
\text { conflict }\end{array}$ & $\begin{array}{l}\text { NTM } \\
\text { conflict }\end{array}$ & $\begin{array}{c}\text { Input } \\
\text { Parameter } \\
\text { conflict }\end{array}$ \\
\hline 1 & $\mathrm{CCO} \& \mathrm{EE}$ & $\checkmark$ & $\checkmark$ & $X$ & $\checkmark$ & $\mathrm{X}$ & $\mathrm{X}$ \\
\hline 2 & MLB \& EE & $X$ & $\checkmark$ & $\mathrm{X}$ & $\checkmark$ & $\mathrm{X}$ & $\mathrm{X}$ \\
\hline 3 & $\begin{array}{l}\text { New eNB/ HeNB/ } \\
\text { Relay \& EE }\end{array}$ & $\mathrm{X}$ & $\mathrm{X}$ & $\mathrm{X}$ & $\checkmark$ & $\checkmark$ & $\mathrm{X}$ \\
\hline 4 & $\mathrm{COC} \& \mathrm{COC}$ & $\checkmark$ & $\checkmark$ & $\mathrm{X}$ & $\checkmark$ & $\mathrm{X}$ & $\mathrm{X}$ \\
\hline 5 & $\begin{array}{l}\text { Two PCl } \\
\text { Instances }\end{array}$ & $\mathrm{X}$ & $\mathrm{X}$ & $\mathrm{X}$ & $\checkmark$ & $\mathrm{X}$ & $\checkmark$ \\
\hline 6 & MRO \& MLB & $\checkmark$ & $\mathrm{X}$ & $\mathrm{X}$ & $\checkmark$ & $\mathrm{X}$ & $\mathrm{X}$ \\
\hline 7 & CCO (RET \& TXP) & $\mathrm{X}$ & $\mathrm{X}$ & $\checkmark$ & $\checkmark$ & $\mathrm{X}$ & $\mathrm{X}$ \\
\hline 8 & $\begin{array}{l}\text { New eNB/ HeNB/ } \\
\text { Relay \& MLB }\end{array}$ & $\mathrm{X}$ & $\mathrm{X}$ & $\mathrm{X}$ & $\checkmark$ & $\checkmark$ & $\mathrm{X}$ \\
\hline 9 & $\mathrm{CCO} \& \mathrm{ICIC}$ & $\checkmark$ & $\checkmark$ & $\mathrm{X}$ & $\checkmark$ & $\mathrm{X}$ & $\mathrm{X}$ \\
\hline 10 & MRO \& COC & $\mathrm{X}$ & $\checkmark$ & $\mathrm{X}$ & $\checkmark$ & $\mathrm{X}$ & $\mathrm{X}$ \\
\hline 11 & COC (RET \& TXP) & $\mathrm{X}$ & $\mathrm{X}$ & $\checkmark$ & $\checkmark$ & $\mathrm{X}$ & $\mathrm{X}$ \\
\hline 12 & $\mathrm{MRO} \& \mathrm{CCO}$ & $\mathrm{X}$ & $\checkmark$ & $\mathrm{X}$ & $\checkmark$ & $\mathrm{X}$ & $\mathrm{X}$ \\
\hline 13 & MLB \& COC & $\mathrm{X}$ & $\checkmark$ & $\mathrm{X}$ & $\checkmark$ & $\mathrm{X}$ & $\mathrm{X}$ \\
\hline 14 & $\begin{array}{l}\text { New eNB/ HeNB/ } \\
\text { Relay \& CCO }\end{array}$ & $\mathrm{X}$ & $\mathrm{X}$ & $\mathrm{X}$ & $\checkmark$ & $\checkmark$ & $\mathrm{X}$ \\
\hline 15 & MRO \& PCI & $\mathrm{X}$ & $\checkmark$ & $\mathrm{X}$ & $\mathrm{X}$ & $\mathrm{X}$ & $\mathrm{X}$ \\
\hline 16 & MLB \& PCI & $\mathrm{X}$ & $\checkmark$ & $\mathrm{X}$ & $\mathrm{X}$ & $\mathrm{X}$ & $\mathrm{X}$ \\
\hline 17 & MLB \& CCO & $\mathrm{X}$ & $\checkmark$ & $\mathrm{X}$ & $\checkmark$ & $\mathrm{X}$ & $\mathrm{X}$ \\
\hline 18 & $\begin{array}{l}\text { New eNB/ HeNB/ } \\
\text { Relay \& MRO }\end{array}$ & $\mathrm{X}$ & $\mathrm{X}$ & $\mathrm{X}$ & $\checkmark$ & $\checkmark$ & $\mathrm{X}$ \\
\hline 19 & $\mathrm{CCO} \& \mathrm{PCl}$ & $\mathrm{X}$ & $\checkmark$ & $\mathrm{X}$ & $\checkmark$ & $\mathrm{X}$ & $\mathrm{X}$ \\
\hline 20 & $\mathrm{COC} \& \mathrm{PCl}$ & $\mathrm{X}$ & $\checkmark$ & $\mathrm{X}$ & $\checkmark$ & $\mathrm{X}$ & $\mathrm{X}$ \\
\hline 21 & $\begin{array}{c}\text { New eNB/ HeNB/ } \\
\text { Relay \& ANR }\end{array}$ & $\mathrm{X}$ & $\mathrm{X}$ & $\mathrm{X}$ & $\checkmark$ & $\sqrt{ }$ & $\mathrm{X}$ \\
\hline 22 & $\begin{array}{c}\text { New eNB/ HeNB/ } \\
\text { Relay \& ICIC }\end{array}$ & $\mathrm{X}$ & $\mathrm{X}$ & $\mathrm{X}$ & $\checkmark$ & $\checkmark$ & $\mathrm{X}$ \\
\hline 23 & MRO \& EE & $\mathrm{X}$ & $\checkmark$ & $\mathrm{X}$ & $\checkmark$ & $\mathrm{X}$ & $\mathrm{X}$ \\
\hline
\end{tabular}




\section{Chain Reaction among SON Function Conflicts or Nested Conflicts:}

Some of the SON function conflicts may occur as a reaction of other SON function conflicts. For example, it is evident from Fig. 1 (a) that MRO and MLB functions conflict is initiated as a chain reaction of MLB and CCO or MLB and EE functions conflicts. We refer to chain reactions among SON functions as "Nested Conflicts", as shown in Fig. 1 (a).

Nested conflicts of SON functions can change the priorities of SON conflict resolution because some low priority conflicts can trigger crucial SON function conflicts. For example, MLB and EE functions conflict is considered as a low priority conflict because this conflict does not affect the end user quality of service. However, MLB and EE conflict can trigger MRO and MLB conflict as a nested conflict which can have severe impact not only on the end user quality of service due to handover problems, such as call drop and RLF, but also on the efficiency of network resource utilization due to ping pong and too late handover problems. Therefore, we can conclude that less important nested SON function conflicts may also deteriorate the end user quality of service and efficiency of radio resource utilization. Moreover, Nested conflicts are more challenging for root cause evaluation of the problem due to chain reaction among multiple SON function conflicts.

\section{HYBRID SELF-COORDINATION FRAMEWORK}

Given the changing and complex nature of SON function conflicts, hybrid Self-Coordination with diverse coordination techniques is essential for conflict resolution among SON functions. We refer to our framework for Self-Coordination solution as hybrid, if it makes use of more than one distinct Self-Coordination mechanism. In this section, we propose such a hybrid SelfCoordination mechanism for conflict resolution between MRO and EE SON functions. This Self-Coordination mechanism paves the way for possible evolution of SON conflict resolution.

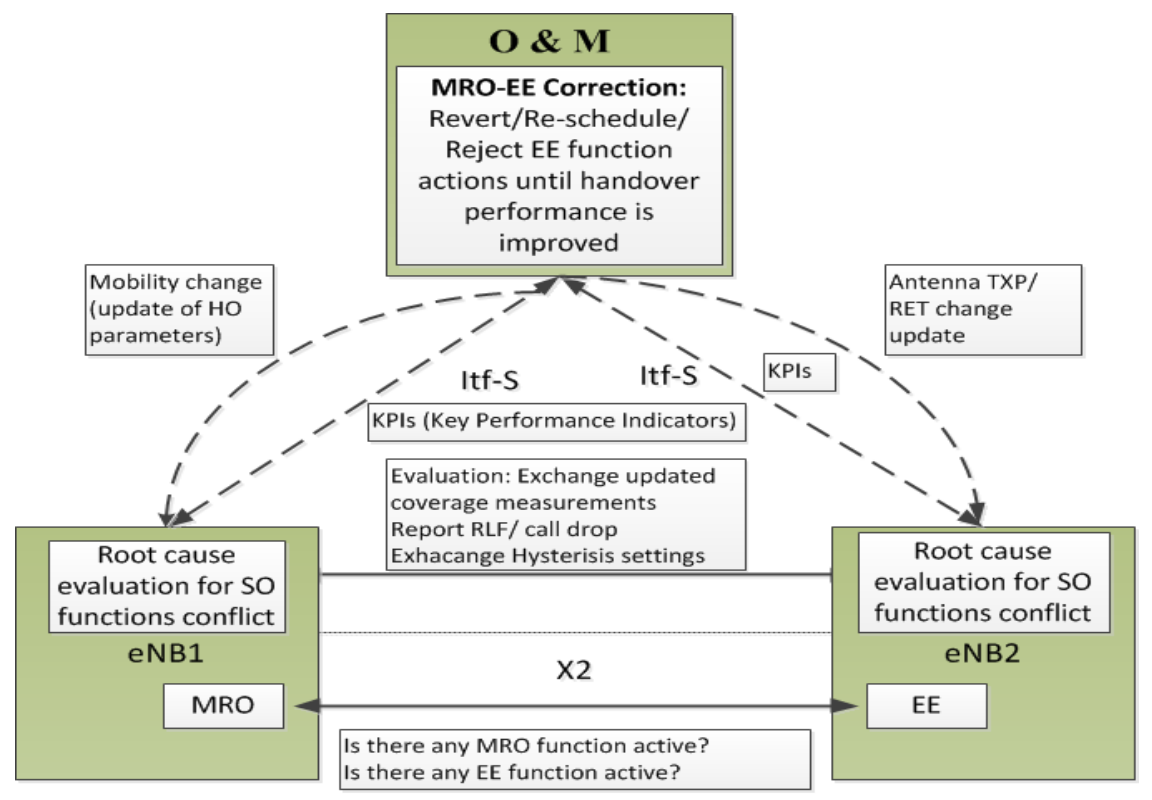




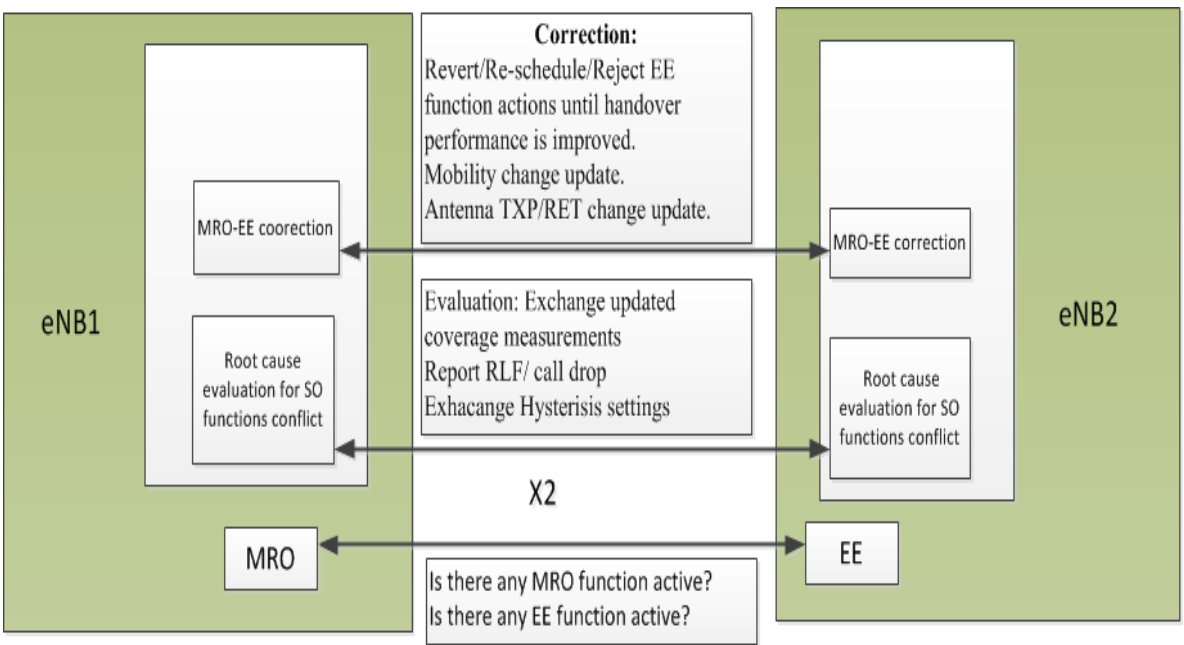

(b) Distributed Architecture

Fig. 3: Hybrid Self-Coordination between MRO \& EE functions

\section{A. Centralised and Distributed Approach for Conflict Resolution among SON Functions:}

A hybrid Self-Coordination mechanism based on centralised architecture for conflict resolution among MRO and EE functions is presented in Fig. 3 (a). More specifically, for conflict free operation of MRO and EE functions, both eNB1 and eNB2 communicate with each other over X2 interface in order to check whether there is any conflicting MRO or EE function active. The rationale behind the proposal of scanning for conflicting SON functions is to re-schedule or delay the execution of the current MRO or EE function until the effects of the previously executing MRO or EE function become visible in the measurement. In this way, measurement conflict between MRO and EE functions can be avoided. However, despite the above Self-Coordination between eNB1 and eNB2, there is still a possibility of conflict between MRO and EE functions due to the logical dependency between these function objectives. In this situation, root cause evaluation procedure is executed at both eNB1 and eNB2 in order to resolve Logical dependency conflict between MRO and EE functions. The eNBs exchange up-to-date coverage measurement with each other in order to measure the accuracy of the handover triggering point. Moreover, the eNB1 and eNB2 exchange root cause evaluation results with Operation and Maintenance (O\&M) server via Itf-S interface. Finally, if the handover problem is caused by EE function actions then O\&M server takes corrective measures. More specifically, the O\&M server conveys new antenna TXP/RET and handover triggering point settings to eNB1 and eNB2 in order to resolve the conflict between MRO and EE functions.

It must be mentioned here that the above Self-Coordination mechanism is hybrid in nature because on one hand it makes use of information exchange between eNB1 and eNB2 in order to avoid Measurement conflict between MRO and EE functions and on 
the other hand it utilizes root cause evaluation procedure in order to resolve the Logical Dependency conflict between MRO and EE functions. The hybrid Self-Coordination mechanism shown in Fig. 3 can be generalized to other SON function conflicts associated with Measurement and Logical Dependency conflict categories listed in TABLE 1. More specifically, the information exchange between neighbouring eNBs about the currently executing SON function can avoid possible measurement conflicts among SON functions and root cause evaluation of network performance degradation problems at eNBs can resolve Logical Dependency conflicts between SON functions.

A hybrid Self-Coordination mechanism based on distributed architecture for conflict resolution among MRO and EE functions is presented in Fig. 3 (b). In distributed Self-Coordination solution, corrective measures for conflict resolution regarding new handover triggering point and antenna TXP are decided at eNB1 and eNB2 instead of O\&M server.

\section{B. Performance Evaluation}

We now evaluate the proposed hybrid Self-Coordination algorithm between MRO and EE SON functions. The network topology we used for performance evaluation consists of seven macro-cellular base stations with uniformly distributed users as shown in Fig. 4(a). We present a graph theory based approach for modeling the network under consideration as shown in Fig. 4(b). In a LTE-Advanced Self-Organising network, we let $\beta$ be a set of macro base stations and $\tau$ be a set of users. The macro base stations and users constitute the vertices of a graph, representing our network. The edges of the graph represent the connectivity opportunities among the vertices.

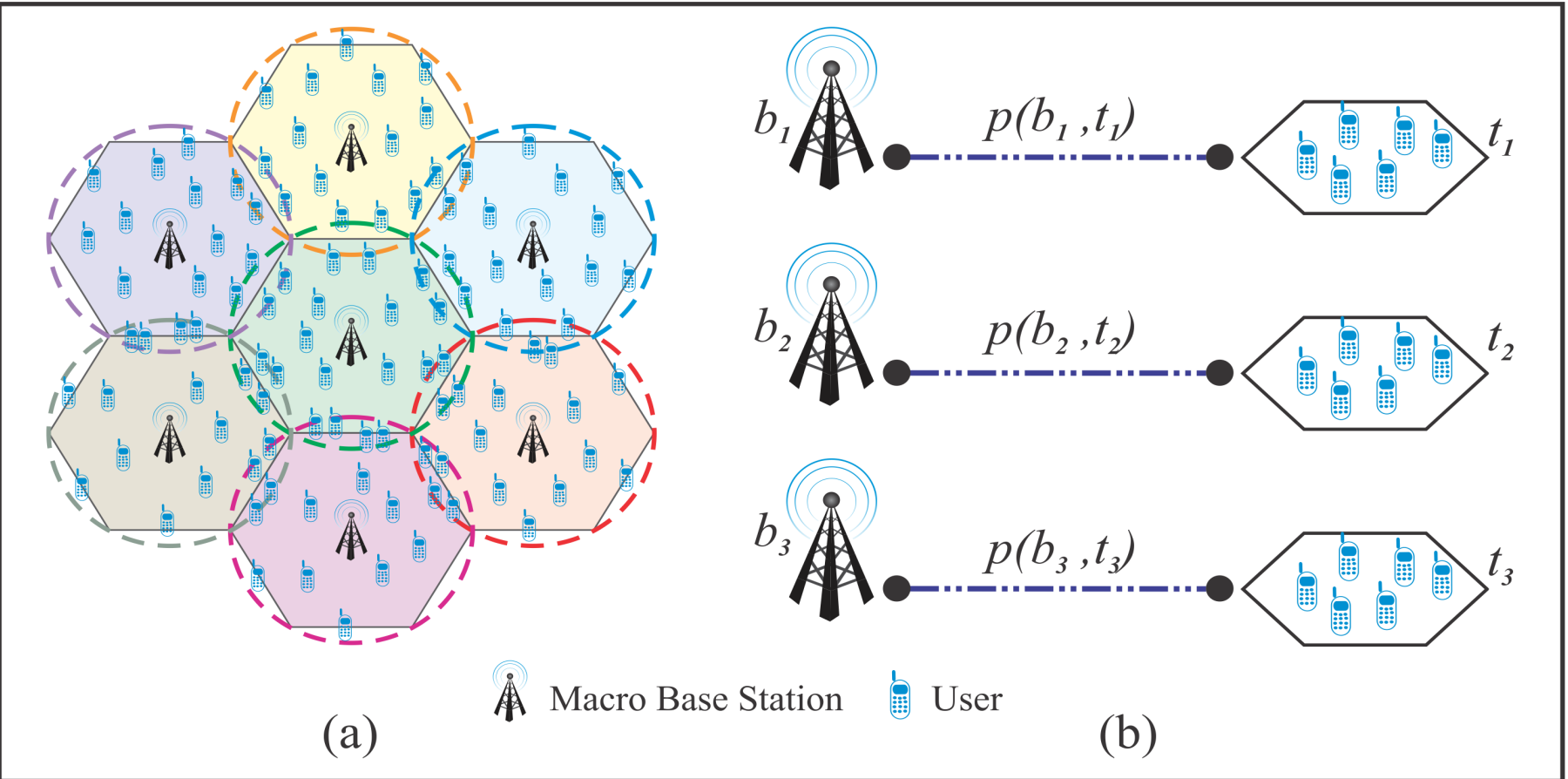

Fig. 4. An illustration of a macro-cellular network topology (a) An overview of a 7 cell macro-cellular network (b) A graph based representation of a macro-cellular network. 
Our objective is not only to minimize the power consumption of the network but also to reduce the handover failures. We formulate the hybrid Self-Coordination algorithm between MRO and EE SON functions as an Integer Linear Programming (ILP) problem. Formally, it can be written as:

$$
\min \sum_{b \in \beta}\left[y_{e e_{b}}\left(\sum_{t \in \tau} y_{e_{e o p t}} p(b, t) x(b, t)\right)+y_{m_{b r} o_{b}} H_{b}\right]
$$

where $y_{e e_{b}}, y_{\text {mro }_{b}} \in\{0,1\}$ are binary variables expressing, respectively, whether MRO and EE functions are active or inactive, $y_{\text {eeopt }_{b}}$ is a real variable which can have values between 0 and 1 in order to optimize transmit power $p(b, t)$ between base station $b$ and user $t, x(b, t)$ represents the data transfer between base station $b$ and user $t$ and $H_{b}$ represents the hysteresis parameter of base station $b$. The binary variables $y_{e e_{b}}$ and $y_{m r o_{b}}$ are utilized to control the execution of conflicting EE and MRO functions in order to avoid Measurement conflicts. Moreover, we apply a lower bound on real variables $y_{e e o p t_{b}}$ such that EE function maintains sufficient coverage overlap with neighbouring cells in order to avoid Logical Dependency conflict between EE and MRO functions. The objective function is linear and can be solved optimally using the state-of-the-art IBM CPLEX Optimizer. Fig. 5(a) depicts a comparison of handover failure ratio for our proposed hybrid Self-Coordination between MRO and EE with uncoordinated MRO and EE SON functions. From the plot, it can be seen that handover failure ratio of hybrid Self-Coordinated MRO and EE SON functions is significantly lower than uncoordinated MRO and EE SON functions.

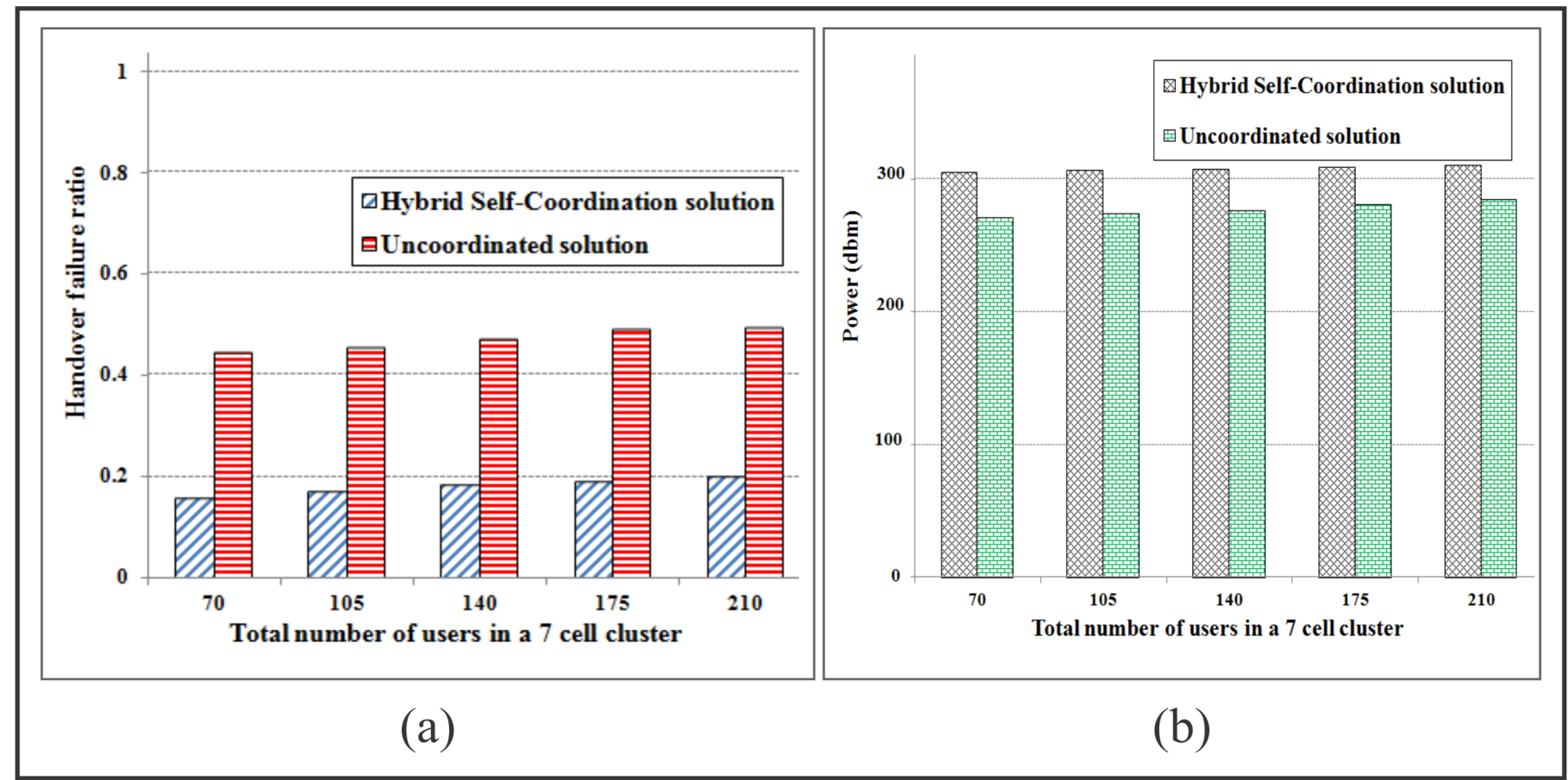

Fig. 5. The performance evaluation of hybrid Self-Coordination between MRO and EE (a) The performance analysis of handover failure ratio (b) The performance evaluation of transmission power consumption. 
A comparison of power consumption for hybrid Self-Coordination between MRO and EE with uncoordinated MRO and EE SON functions is shown in Fig. 5(b). Fig. 5(b) shows that the transmit power consumption of the hybrid Self-Coordination solution is slightly higher than the uncoordinated solution. The rationale behind this fact is that hybrid Self-Coordination solution provides better coverage than an uncoordinated solution in order to avoid severe effects on the end user quality of service, such as handover failures.

\section{CONCLuSion AND Future Work}

While Self-Organising Network (SON) has attracted significant attention from industry, the most challenging issue in implementing SON in reality is the identification of the best possible Self-Coordination mechanisms among conflicting SON functions in order to guarantee a stable and desired network operation. For in depth modelling and rectification of conflicting SON functions, we have presented a hybrid classification of SON function conflicts, which classifies these conflicts on the basis of soft and hard classification and paves the way for designing appropriate Self-Coordination solutions. More specifically, for comprehensive investigation of SON function conflicts, we presented a case study of Mobility Load Balancing (MLB), Mobility Robustness Optimisation (MRO), Coverage and Capacity Optimisation (CCO) and Energy efficiency (EE) SON function conflicts using Reference Signal Received Power (RSRP) plots in multi-cell environments and 3GPP architecture details. In this direction, we have also proposed an analytical mapping of conventional cellular networks' spectral efficiency and energy efficiency performance metrics trade-off to SON CCO and EE SON functions conflict. We have proposed that SON function conflicts associated with Output Parameter, Input Parameter, Network Topology Mutation, Logical Dependency and Key Performance Indicator conflict categories can also be classified into Measurement conflict category. Moreover, we have identified the case of SON function conflicts triggering more conflicts. More specifically, we have shown that a low priority conflict between MLB and CCO functions triggered a crucial conflict between MRO and MLB functions. For possible evolution of the Self-Coordination paradigm, we have proposed both centralised and distributed hybrid Self-Coordination mechanisms between MRO and EE functions. We have presented the performance evaluation of the hybrid Self-Coordination solution using a graph theory based approach. The performance evaluation shows that the handover failure ratio of the proposed hybrid SelfCoordination between MRO and EE functions is significantly lower than the uncoordinated execution of MRO and EE functions. We have recommended that scanning for possible conflicting SON functions using the information exchange among neighbouring eNBs over X2 interface can avoid Measurement conflicts. Moreover, the proposed hybrid Self-Coordination mechanism can be generalized to other SON function conflicts associated with Measurement and Logical Dependency conflict categories. Root cause evaluation of nested conflicts which arise due to the chain reaction among multiple SON function conflicts is still an open area of research. The hybrid classification of SON function conflicts and Self-Coordination mechanisms 
presented in this paper will assist further research for designing conflict-free SON solutions, which will eventually expedite the incorporation of SON in next generation wireless communications systems.

\section{ACKNOWLEDGMENT}

This work was made possible by NPRP grant No. 5-1047-2-437 from the Qatar National Research Fund (a member of The Qatar Foundation). The statements made herein are solely the responsibility of the authors.

\section{REFERENCES}

[1] NGMN, "Recommendation on SON and O\&M Requirements," Requirement Specification, www.ngmn.org, version 1.23, 2008.

[2] Aliu, O.G.; Imran, A.; Imran, M.A.; Evans, B., "A Survey of Self Organisation in Future Cellular Networks," Communications Surveys \& Tutorials, IEEE, vol.15, no.1, pp.336,361, First Quarter 2013

[3] M. Peng, D. Liang, Y. Wei, J. Li, and H. H. Chen, "Self-Configuration and Self-Optimization in LTE-Advanced Heterogeneous Networks," IEEE Communications Magazine, vol. 51, no. 5, pp. 36-45, 2013.

[4] 3GPP "Telecommunication Management; Self-Organizing Networks (SON); Concepts and Requirements (Release 11)," 3GPP TS 32.500, v. 11.1.0, 2011-12. http://www.3gpp.org/ftp/Specs/html-info/32500.htm.

[5] T. Jansen, I. Balan, J. Turk, I. Moerman, and T. Kurner, "Handover Parameter Optimization in LTE Self-Organizing Networks", In Proceedings of IEEE Vehicular Technology Conference (VTC Fall 2010), pp. 1 -5, 2010.

[6] T. Jansen, M. Amirijoo, U. Turke, L. Jorguseski, K. Zetterberg, R. Nascimento, L.C. Schmelz, J. Turk, and I. Balan, "Embedding multiple self organisation functionalities in future radio access networks," In Proceedings of IEEE Vehicular Technology Conference (VTC Spring 2009), pp. 1 -5, 2009.

[7] www.fp7-socrates.eu.

[8] S. Hamalainen, C. Sartori, Henning Sanneck, "LTE Self-Organising Networks (SON): Network Management Automation for Operational Efficiency," WILEY, 2011.

[9] L.C. Schmelz, M. Amirijoo, A. Eisenblaetter, R. Litjens, M. Neuland, and J. Turk,"A Coordination Framework for SelfOrganisation in LTE Networks," In Proceedings of IEEE Integrated Network Management (IM' 2011), pp. 193 -200, 2011.

[10] H. Y. Lateef, A. Imran, A. Abu-Dayya, "A Framework for Classification of Self-Organising Network Conflicts and Coordination Algorithms," In Proceedings of IEEE Personal Indoor and Mobile Radio Communications (PIMRC' 2013), pp. 2913 -2918, 2013. www.qson.org/project-publications

[11] Chen, Yan, et al. "Fundamental Trade-offs on Green Wireless Networks", IEEE Communications Magazine, vol. 49, no. 6, pp. 30-37, 2011.

[12] O. Oluwakayode, F. Heliot, and M. A. Imran, "On the Energy Efficiency-Spectral Efficiency Trade-off in the Uplink of Comp System." IEEE Transactions on Wireless Communications, vol. 11, no. 2, pp. 556-561, 2012.

[13] 3GPP Tech. Spec. Group, E-UTRA, “LTE; Evolved Universal Terrestrial Radio Access (E-UTRA); Radio Resource Control (RRC); Protocol specification,” 3GPP TS 36.331, v. 11.3.0, 2013-04. http://www.3gpp.org/ftp/Specs/html-info/36331.htm

[14] L. Yun, L. Man, C. Bin, W. Yong, and L. Wenjing, "Dynamic Optimization of Handover Parameters Adjustment for Conflict Avoidance in Long Term Evolution," Communications, China, vol. 10, no. 1, pp. 56-71, 2013.

[15] L. Yun, L. Man, C. Bin, W. Yong, and L. Wenjing, "A conflict avoid method between load balancing and Mobility Robustness Optimization in LTE,” In Proceedings of IEEE Communications in China (ICCC' 2012), pp. 143 -148, 2012. 